\title{
Outage Analysis of Cognitive Hybrid Satellite-Terrestrial Networks with Hardware Impairments and Multi-Primary Users
}

\author{
Kefeng Guo, Student Member, IEEE, Kang An, Bangning Zhang, Member, IEEE, \\ Yuzhen Huang, Member, IEEE, Gan Zheng, Senior Member, IEEE
}

\begin{abstract}
This paper investigates the effects of practical hardware impairments (HIs) on a cognitive hybrid satellite-terrestrial networks (CHSTN) with multiple primary users (PUs). The widely-employed Shadowed-Rician fading distribution is adopted to model the satellite-terrestrial channel. CHSTN can provide comprehensive wireless coverage as well as enhanced spectrum resource usage by considering the requirements of both spectrum efficiency and reliability. Specifically, we derive the closed-form expression of the outage probability (OP) for the considered system in the presence of interference power constraints imposed by multiple adjacent terrestrial PUs. To gain further insights at high signal-to-noise ratios (SNRs), the asymptotic expression for the OP is also derived. Numerical results confirm the correctness and effectiveness of our performance analysis.
\end{abstract}

Index Terms-Cognitive hybrid satellite-terrestrial networks (CHSTN), multi-primary users, hardware impairments (HIs).

\section{INTRODUCTION}

$\mathbf{T}$ HE increasing demands for high data rate and seamless coverage have been viewed as the major challenge of the future wireless networks. On this front, hybrid satelliteterrestrial networks (HSTN) have been considered as an attractive and promising infrastructure, which can provide ubiquitous coverage, higher transmission rate and broadcast/multicast reliable service to portable and mobile users [1]. Until now, researches have focused on the HSTN from various performance metrics, such as outage probability (OP), ergodic capacity, symbol error rate, throughput in single-antenna scenarios, where terrestrial relay cooperation was employed to assist the satellite mobile networks. Besides, extension works to multi-antenna scenarios with orthogonal space-time block coding (OSTBC), and beamforming (BF) schemes were analyzed in [2] and [3], respectively.

This work was supported by the National Science Foundation of China (No. 61401508), and (No. 61471392), the Jiangsu Provincial Natural Science Foundation of China (No.20150719) and the work of G. Zheng was supported by the UK EPSRC under grant EP/N007840/1, and the Leverhulme Trust under grant number RPG-2017-129.

K. Guo and B. Zhang are with the College of Communications Engineering, Army Engineering University of PLA, Nanjing, China (e-mail: guokefeng.cool@163.com; bangning_zhang@sina.com.

K. An is with the National University of Defense Technology, Nanjing, China (e-mail:ankang@nuaa.edu.cn).

Y. Huang is with the Artificial Intelligence Research Center, National Innovation Institute of Defense Technology, Beijing, China (e-mail: yzh_huang@sina.com).

G. Zheng is with the Wolfson School of Mechanical, Electrical, and Manufacturing Engineering, Loughborough University, Loughborough LE11 3TU, U.K. (e-mail: g.zheng @lboro.ac.uk)
Recently, cognitive ratio (CR) has received much interest in satellite communications (SatComs) due to the ability of alleviating the spectrum shortage problem, especially when the growing number of satellite services is consuming the already limited spectral resources [4], [5]. The integration of CR into HSTN can offers the advantages of not only increasing the overall spectral efficiency by spectrum sharing but also extending the wireless coverage through the deployment of relays, and constitute a cognitive hybrid satellite-terrestrial network (CHSTN) architecture [6]-[12].

However, in practice the hardware nodes in wireless networks are not perfect for various practical reasons, such as amplifier non-linearities, I/Q-imbalance and phase noise [13], [14]. In [15], the general hardware impairments (HIs) model was proposed for the relay network. In [16], [17], the authors investigated impact of HIs on the system performance for the cognitive terrestrial networks.

However, the effect of HIs on the CHSTN is not understood, which is necessary in the satellite-terrestrial networks. Thus this letter investigates the CHSTN with HIs and interference power constraints imposed by multi-primary users (PUs). Particularly, the exact and asymptotic expressions for the OP are derived. To the best of our knowledge, this is the first time these theoretical results have been obtained, which are useful to analyze the effect of non-ideal circuit HIs on the performance of CHSTN. Numerical simulation results are provided to validate the performance analysis.

\section{System Model and Problem Formulation}

We consider the CHSTN, which consists of a secondary satellite source (S) communicating with a secondary destination (D) with the assistance of the terrestrial relay (R) with $N$ antennas in the presence of $M$ terrestrial primary users (PUs) with single antenna. $S$ and $D$ are equipped with single antenna, respectively. As the reasons provided above, all the nodes in the networks suffer from HIs. We assume that there is no direct link between $\mathrm{S}$ and $\mathrm{D}$ due to the rain, fog, or heavy shadowing [6]. The terrestrial $\mathrm{R}$ works in the half-duplex mode with the decode-and-forward (DF) protocol. Hence, $\mathrm{S}$ takes two time slots to communicate with D. In the first time slot, S sends its signal $s(t)$ with $E\left[|s(t)|^{2}\right]=1$ to R, where $E[\cdot]$ denotes 
expectation. The signal received at $\mathrm{R}$ is given by

$$
y_{R}(t)=\mathbf{h}_{S R} \mathbf{w}_{S R}^{H}\left[\sqrt{P_{S}} s(t)+\eta_{S R}(t)\right]+\mathbf{w}_{S R}^{H} \mathbf{n}_{R}(t),
$$

where $\mathbf{h}_{S R}$ is the channel vector between $\mathrm{S}$ and $\mathrm{R}$ modeled by the shadowed-Rician (SR) fading, $\mathbf{w}_{S R}^{H}$ the receive $\mathrm{BF}$ weight vector with $\left\|\mathbf{w}_{S R}\right\|^{2}=1,(\cdot)^{H}$ the conjugate transpose. $P_{S}$ is the transmit power from $\mathrm{S}, \eta_{S R}(t)$ the distortion noise caused by HIs which is present as $\eta_{S R}(t) \sim \mathcal{C N}\left(0, k_{S R}^{2} P_{S}\right)$, where $\mathcal{C N}\left(0, k_{S R}^{2} P_{S}\right)$ is the complex Gaussian distribution of a random vector 0 and covariance $k_{S R}^{2} P_{S}, k_{S R}$ represents the HIs level [18]. $\mathbf{n}_{R}(t)$ is the additive white Gaussian noise (AWGN) at R, which is an $N \times 1$ AWGN vector whose distribution is $\mathcal{C N}\left(0, \delta_{R}^{2} \mathbf{I}_{N \times 1}\right)$.

In the second time slot, $\mathrm{R}$ forwards the received signal to $\mathrm{D}$ with the DF protocol, and the received signal is given by

$$
y_{D}(t)=\mathbf{w}_{R D}^{H} \mathbf{h}_{R D}\left[\sqrt{P_{R}} s(t)+\eta_{R D}(t)\right]+n_{D}(t),
$$

where $\mathbf{w}_{R D}^{H}$ is the transmit $\mathrm{BF}$ vector at $\mathrm{R}$ with $\left\|\mathbf{w}_{R D}\right\|^{2}=1$, $\mathbf{h}_{R D}$ the channel coefficient vector between $R$ and $D$ modeled as Rayleigh fading, $P_{R}$ the transmit power of $\mathrm{R}, \eta_{R D}(t)$ the distortion noise caused by HIs which is distributed as $\eta_{R D}(t) \sim \mathcal{C N}\left(0, k_{R D}^{2} P_{R}\right), k_{R D}$ presents the HIs level. $\mathbf{n}_{D}(t)$ is the AWGN at D distributed as $\mathbf{n}_{D} \sim \mathcal{C N}\left(0, \delta_{D}^{2}\right)$. When maximum ratio combining (MRC) and maximum ratio transmitting (MRT) are used by the system, we can get $\mathbf{w}_{S R}=\frac{\mathbf{h}_{S R}}{\left\|\mathbf{h}_{S R}\right\|}$ and $\mathbf{w}_{R D}=\frac{\mathbf{h}_{R D}}{\left\|\mathbf{h}_{R D}\right\|}$.

Hereby, the transmit powers of $P_{S}$ and $P_{R}$ are constrained to limit interference at PUs to be below maximum tolerable interference power $Q$. It follows that the received interference powers at PUs from $S$ and $R$ must be constrained, i.e, $E\left[P_{S} \sum_{p=1}^{M}\left|h_{S P_{p}}\right|^{2}\left(1+k_{S P_{P}}^{2}\right)\right] \leq Q$ and $E\left[P_{R} \sum_{r=1}^{M}\left|\mathbf{w}_{R D}^{H} \mathbf{h}_{R P_{r}}\right|^{2}\left(1+k_{R P_{r}}^{2}\right)\right] \leq Q,{ }^{1}$ according to [15], $k_{S P_{p}}$ and $k_{R P_{r}}$ denotes the aggregate level of HIs of the $p$-th PU link and $r$-th PU link, respectively. Consequently, we have [19] $P_{S}=Q /\left[\sum_{p=1}^{M}\left|h_{S P_{p}}\right|^{2}\left(1+k_{S P_{p}}^{2}\right)\right]$ and $P_{R}=Q /\left[\sum_{r=1}^{M}\left|h_{R P_{r}}\right|^{2}\left(1+k_{R P_{r}}^{2}\right)\right]$.

We assume that each PU channel is independently and identically distributed (i.i.d) with the same channel parameters. The HIs levels of them are assumed same [15]. Hence, from (1) and (2), by using $P_{S}$ and $P_{R}$, the signal-to-noise-anddistortion-ratio (SNDR) at R and D are expressed as

$$
\begin{gathered}
\gamma_{R}=\gamma_{S R} Q /\left[\gamma_{S R} Q k_{S R}^{2}+\gamma_{S P}\left(1+k_{S P}^{2}\right) \delta_{R}^{2}\right], \\
\gamma_{D}=\gamma_{R D} Q /\left[\gamma_{R D} Q k_{R D}^{2}+\gamma_{R P}\left(1+k_{R P}^{2}\right) \delta_{D}^{2}\right],
\end{gathered}
$$

where $\gamma_{S R}=Q\left\|\mathbf{h}_{S R}\right\|^{2} / \delta_{R}^{2}, \gamma_{S P}=Q \sum_{p=1}^{M}\left|h_{S P_{p}}\right|^{2} / \delta_{R}^{2}$,

${ }^{1}$ We consider that the PUs are located in close proximity to each other with the same fading severity parameter and average powers under the same satellite beam radius. This is the worse-case scenario instead of the distributed located PUs, where the maximum interference link is selected to guarantee the operation of primary network. Noteworthy, the adopted interference constraint is a stricter one than those in existing works.
$k_{S P_{1}}=k_{S P_{2}}=\cdots k_{S P_{M}}=k_{S P}, \gamma_{R D}=Q\left|\mathbf{h}_{R D}\right|^{2} / \delta_{D}^{2}$,
$\gamma_{R P}=Q \sum_{r=1}^{M}\left|h_{R P_{r}}\right|^{2} / \delta_{D}^{2}, k_{R P_{1}}=k_{R P_{2}}=\cdots k_{R P_{M}}=k_{R P}$.

As the DF protocol is used by $\mathrm{R}$, the final SNDR of the system is given by

$$
\gamma_{e}=\min \left(\gamma_{R}, \gamma_{D}\right)
$$

By assuming MRC is used by PUs and setting $\sum_{p=1}^{M}\left|h_{S P_{p}}\right|^{2}=$ $\left|\mathbf{w}_{S P}^{H} \mathbf{h}_{S P}\right|^{2}$ with $\mathbf{w}_{S P}=\frac{\mathbf{h}_{S P}}{\left\|\mathbf{h}_{S P}\right\|}$, then we can get

$$
\mathbf{h}_{S J}=F_{S J} \mathbf{g}_{S J}, J \in\{R, P\},
$$

where $\mathrm{g}_{S J}$ is the channel coefficient vector, which is often assumed to undergo SR fading [6]-[8], and $F_{S J}$ a scaling parameter including many practical effects, such as free space loss (FSL) and antenna pattern, which is given by $F_{S J}=$ $C \sqrt{G_{t, S J} G_{r, S J}} /\left(4 \pi f d_{S J} \sqrt{K_{B} T B}\right)$ with $C$ being the light speed, $f$ the carrier frequency, $d_{S J}$ the distance between the satellite and the user, $K_{B}=1.38 \times 10^{-23} \mathrm{~J} / K$ the Boltzman constant, $T$ the receiver noise temperature, and $B$ the carrier bandwidth. Meanwhile, $G_{r, S J}$ denotes the receive gain, and $G_{t, S J}$ the satellite beam gain, which can be approximately written as $G_{t, S J}=G_{\max }\left(\frac{J_{1}(u)}{2 u}+36 \frac{J_{3}(u)}{u^{3}}\right)^{2}$ [20], with $\mathrm{G}_{\max }$ being the maximal beam gain and $u=2.07123 \frac{\sin \varphi}{\sin \varphi_{3 \mathrm{~dB}}}$, where $\varphi$ is the angle between the location of the corresponding receiver and the beam center with respect to the satellite, and $\varphi_{3 \mathrm{~dB}}$ is the $3-\mathrm{dB}$ angle.

Hence, $\gamma_{S R}$ and $\gamma_{S P}$ are re-written as

$$
\begin{aligned}
& \gamma_{S R}=Q F_{S R}^{2}\left\|\mathbf{g}_{S R}\right\|^{2} / \delta_{R}^{2} \triangleq \bar{\gamma}_{S R}\left\|\mathbf{g}_{S R}\right\|^{2}, \\
& \gamma_{S P}=Q F_{S P}^{2}\left\|\mathbf{g}_{S P}\right\|^{2} / \delta_{R}^{2} \triangleq \bar{\gamma}_{S P}\left\|\mathbf{g}_{S P}\right\|^{2},
\end{aligned}
$$

where $\bar{\gamma}_{S R}=Q F_{S R}^{2} / \delta_{R}^{2}$ is the average SNR of the $\mathrm{S}$ to the $\mathrm{R}$ link and $\bar{\gamma}_{S P}=Q F_{S P}^{2} / \delta_{R}^{2}$ that of the $\mathrm{S}$ to PUs link.

Remark 1. It is worth-mentioning that we consider a more general case of CHSTN with multi-PUs and HIs, where practical propagation factors, such as satellite beam pattern and path loss, are taken into account. Thus, our work includes the system model in [6], [9] as a special case, where only one $P U$ and ideal hardware is assumed and the scaling parameter in (6) is reduced to $F_{S J}=1$. Besides, our work extends the work of [21], when ideal hardware is considered.

\section{Performance Analysis}

Before analyzing the performance of considered network, we first study the statistical property of the satellite link. In the PUs' link, we consider the worst condition. By letting $U=\{S R, S P\}$, hence the probability distribution function (PDF) of $U$ is given by [22]:

$f_{\gamma_{U}}\left(\gamma_{U}\right)=\sum_{\xi_{1}=0}^{m_{U}-1} \cdots \sum_{\xi_{N}=0}^{m_{U}-1} \Xi\left(N_{U}\right) \gamma_{U}^{\Lambda_{U}-1} e^{-\Delta_{U} \gamma_{U}}, N_{U} \in\{N, M\}$

where

$$
\Xi(N) \triangleq \prod_{\tau=1}^{N} \zeta\left(\xi_{\tau}\right) \alpha_{U}^{N} \prod_{v=1}^{N-1} B\left(\sum_{l=1}^{v} \xi_{l}+v, \xi_{v+1}+1\right),
$$


$\Lambda_{U} \triangleq \sum_{\tau=1}^{N} \xi_{\tau}+N, \zeta\left(\xi_{\tau}\right)=\frac{\left(1-m_{U}\right)_{\xi_{\tau}}\left(-\delta_{U}\right)^{\xi_{\tau}}}{\left(\xi_{\tau} !\right)^{2}\left(\bar{\gamma}_{U}\right)^{\xi_{\tau}+1}}, \Delta_{U}=\frac{\beta_{U}-\delta_{U}}{\bar{\gamma}_{U}}$, $\alpha_{U} \triangleq \frac{\left(\frac{2 b_{U} m_{U}}{2 b_{U} m_{U}+\Omega_{U}}\right)^{m_{U}}}{2 b_{U}}, \beta_{U} \triangleq \frac{1}{2 b_{U}}, \delta_{U} \triangleq \frac{\Omega_{U}}{2 b_{U}\left(2 b_{U} m_{U}+\Omega_{U}\right)}$ and $B(.,$.$) denotes the Beta function [23]. \Omega_{U}, 2 b_{U}$ and $m_{U} \geq 0$ denote the average power of the line of sight (LOS) component, the average power of the multi-path component, and the fading severity parameter ranging from 0 to $\infty$, respectively.

Based on (8) along with integral computation, the cumulative distribution function (CDF) of $\gamma_{U}$ is given by

$$
\begin{aligned}
& F_{\gamma_{U}}\left(\gamma_{U}\right)=1-\sum_{\xi_{1}=0}^{m_{U}-1} \cdots \sum_{\xi_{N}=0}^{m_{U}-1} \sum_{m=0}^{\Lambda_{U}-1} \frac{\Xi(N)\left(\Lambda_{U}-1\right) ! \gamma_{U}^{m}}{m ! \Delta_{U}^{\Lambda-m}} e \\
& \text { In PUs' link, we consider the worst condition. From }[24 \\
& \text { by setting } L=\{R D, R P\} \text {, we can derive the PDF for } \gamma_{R} \\
& \text { and } \gamma_{R P}, \text { respectively, as } \\
& f_{\gamma_{L}}\left(\gamma_{L}\right)=\sum_{i=1}^{\rho\left(\mathrm{A}_{L}\right)} \sum_{j=1}^{\tau_{i}\left(\mathrm{~A}_{L}\right)} \chi_{i, j}\left(\mathrm{~A}_{L}\right) \frac{\mu_{\langle i\rangle}^{-j}}{(j-1) !} \gamma_{L}^{j-1} e^{-\gamma_{L} / \mu_{\langle i\rangle}} \text {, }
\end{aligned}
$$

where $\mathrm{A}_{L}=\operatorname{diag}\left(\mu_{1}, \mu_{1}, \ldots, \mu_{J}\right), J \in\{N, N \times M\}$, $\rho\left(\mathrm{A}_{L}\right)$ is the number of distinct diagonal elements of $\mathrm{A}_{L}$, $\mu_{\langle 1\rangle}>\mu_{\langle 2\rangle}>\ldots>\mu_{\left\langle\rho\left(\mathrm{A}_{L}\right)\right\rangle}$ are the multiplicity of $\mu_{\langle i\rangle}$, and $\chi_{i, j}\left(\mathrm{~A}_{L}\right)$ is the $(i, j)$-th characteristic coefficient of $\mathrm{A}_{L}$ [24].

Similarly, the CDF of $\gamma_{L}$ is given by

$$
F_{\gamma_{L}}\left(\gamma_{L}\right)=1-\sum_{i=1}^{\rho\left(\mathrm{A}_{L}\right)} \sum_{j=1}^{\tau_{i}\left(\mathrm{~A}_{L}\right)} \chi_{i, j}\left(\mathrm{~A}_{L}\right) \sum_{m=0}^{j-1} \frac{1}{m !}\left(\frac{\gamma_{L}}{\mu_{\langle i\rangle}}\right)^{m} e^{-\frac{\gamma_{L}}{\mu_{\langle i\rangle}}} .
$$

\section{A. $O P$}

According to [15], the OP is defined as the SNDR falls below a predefined threshold $\gamma_{0}$, which can be presented as

$$
\begin{aligned}
& P_{\text {out }}\left(\gamma_{0}\right)=\operatorname{Pr}\left(\gamma_{e} \leq \gamma_{0}\right)=\operatorname{Pr}\left[\min \left(\gamma_{R}, \gamma_{D}\right) \leq \gamma_{0}\right] \\
& =\operatorname{Pr}\left(\gamma_{R} \leq \gamma_{0}\right)+\operatorname{Pr}\left(\gamma_{D} \leq \gamma_{0}\right)-\operatorname{Pr}\left(\gamma_{R} \leq \gamma_{0}\right) \operatorname{Pr}\left(\gamma_{D} \leq \gamma_{0}\right) .
\end{aligned}
$$

Next, $\operatorname{Pr}\left(\gamma_{R} \leq \gamma_{0}\right)$ and $\operatorname{Pr}\left(\gamma_{D} \leq \gamma_{0}\right)$ are derived as follows.

First of all, we focus on $\operatorname{Pr}\left(\gamma_{R} \leq \gamma_{0}\right)$. From (3) and (4), when $\gamma_{0}<1 / k_{S R}^{2}$, we can get

$$
\begin{aligned}
& \operatorname{Pr}\left(\gamma_{R} \leq \gamma_{0}\right)=\operatorname{Pr}\left(\frac{\gamma_{S R} Q}{\gamma_{S R} Q k_{S R}^{2}+\gamma_{S P}\left(1+k_{S P}^{2}\right) \delta_{R}^{2}} \leq \gamma_{0}\right) \\
& =\int_{0}^{\infty} F_{\gamma_{S R}}\left(\gamma_{0} \delta_{R}^{2}\left(1+k_{S P}^{2}\right) y /\left[Q\left(1-k_{S R}^{2} \gamma_{0}\right)\right]\right) f_{\gamma_{S P}}(y) d y .
\end{aligned}
$$

Then substituting (8) and (9) into (13), we obtain

$$
\begin{aligned}
& \operatorname{Pr}\left(\gamma_{R} \leq \gamma_{0}\right)=\sum_{\xi_{1}=0}^{m_{S P}-1} \cdots \sum_{\xi_{M}=0}^{m_{S P}-1} \Xi(M)\left[\left(\Lambda_{S P}-1\right) ! \Delta_{S P}^{-\Lambda_{S P}}\right. \\
& -\sum_{\xi_{1}=0}^{m_{S R}-1} \cdots \sum_{\xi_{N}=0}^{m_{S R}-1} \sum_{m=0}^{\Lambda_{S R}-1} \frac{\Xi(N)\left(\Lambda_{S R}-1\right) ! A^{m}}{m ! \Delta_{S R}^{\Lambda_{S R}-m}} \\
& \left.\times\left(\Lambda_{S P}-1+m\right) !\left(\Delta_{S P}+\Delta_{S R} A\right)^{\Lambda_{S P}+m}\right]
\end{aligned}
$$

where $A=\gamma_{0} \delta_{R}^{2}\left(1+k_{S P}^{2}\right) /\left[Q\left(1-k_{S R}^{2} \gamma_{0}\right)\right]$.
Then, by the same manner, when $\gamma_{0}<1 / k_{R D}^{2}$, $\operatorname{Pr}\left(\gamma_{D} \leq \gamma_{0}\right)$ is given by

$$
\begin{aligned}
& \operatorname{Pr}\left(\gamma_{D} \leq \gamma_{0}\right)=\operatorname{Pr}\left(\frac{\gamma_{R D} Q}{\gamma_{R D} Q k_{R D}^{2}+\gamma_{R P}\left(1+k_{R P}^{2}\right) \delta_{D}^{2}} \leq \gamma_{0}\right) \\
& =\int_{0}^{\infty} F_{\gamma_{R D}}\left(\gamma_{0} \delta_{D}^{2}\left(1+k_{R P}^{2}\right) y /\left[Q\left(1-k_{R D}^{2} \gamma_{0}\right)\right]\right) f_{\gamma_{R P}}(y) d y .
\end{aligned}
$$

By substituting (10) and (11) into (15), we can obtain

$$
\begin{aligned}
& \operatorname{Pr}\left(\gamma_{D} \leq \gamma_{0}\right) \\
& =\sum_{i i=1}^{\rho\left(\mathrm{A}_{R P}\right)} \sum_{j j=1}^{\tau_{i}\left(\mathrm{~A}_{R P}\right)} \chi_{i i, j j}\left(\mathrm{~A}_{R P}\right) \frac{\mu_{\langle i i\rangle}^{-j j}}{(j j-1) !}\left[(j j-1) ! \mu_{\langle i i\rangle}^{j j}\right. \\
& -\sum_{i=1}^{\rho\left(\mathrm{A}_{R D}\right)} \sum_{j=1}^{\tau_{i}\left(\mathrm{~A}_{R D}\right)} \sum_{m=0}^{j-1} \chi_{i, j}\left(\mathrm{~A}_{R D}\right) \frac{1}{m !}\left(\frac{B}{\mu_{\langle i\rangle}}\right)^{m} \\
& \left.\times(j j-1+m) !\left(B / \mu_{\langle i\rangle}+1 / \mu_{\langle i i\rangle}\right)^{j+m}\right]
\end{aligned}
$$

where $B=\gamma_{0} \delta_{D}^{2}\left(1+k_{R P}^{2}\right) /\left[Q\left(1-k_{R D}^{2} \gamma_{0}\right)\right]$.

Finally, by taking (14) and (16) into (12), the final expression for OP is derived. In order to save the length of page, here the final expression is omitted.

\section{B. Asymptotic $O P$}

Recalling (8) and (9), when $\bar{\gamma}_{S R}$ is large, they can be written as

$$
F_{\gamma_{S R}}\left(\gamma_{S R}\right)=\frac{\alpha_{S R}^{N}}{N !}\left(\frac{\gamma_{S R}}{\bar{\gamma}_{S R}}\right)^{N} .
$$

Then substituting (17) and (8) into (13), we can get

$$
\begin{aligned}
& \operatorname{Pr}\left(\gamma_{R} \leq \gamma_{0}\right) \\
& =\frac{\alpha_{S R}^{N} A^{N}}{N ! \bar{\gamma}_{S R}^{N}} \sum_{\xi_{1}=0}^{m_{S P}-1} \cdots \sum_{\xi_{M}=0}^{m_{S P}-1} \Xi(M)\left(\Lambda_{S P}-1+N\right) ! \Delta_{S P}^{\Lambda_{S P}+N} .
\end{aligned}
$$

Using the same manner and assuming that each channel has the same channel parameters, we can get the CDF of $\gamma_{R D}$ at high SNRs and the PDF of $\gamma_{R P}$ as, respectively

$$
F_{\gamma_{R D}}\left(\gamma_{R D}\right)=\left(\gamma_{R D} / \bar{\gamma}_{R D}\right)^{N} / N !
$$

and

$$
f_{\gamma_{R P}}\left(\gamma_{R P}\right)=\gamma_{R P}^{N M-1} /\left[(N M-1) ! \bar{\gamma}_{R P}^{N M}\right]
$$

Next, by taking (20) and (19) into (15), we can obtain

$$
\operatorname{Pr}\left(\gamma_{D} \leq \gamma_{0}\right)=\frac{(N+N \times M-1) ! B^{N} \bar{\gamma}_{R P}^{N}}{N !(N \times M-1) ! \bar{\gamma}_{R D}^{N}} .
$$

Furthermore, substituting (18) and (21) into (12), the asymptotic expression of OP can be derived.

Finally, if we set $\bar{\gamma}_{S R}=\bar{\gamma}_{S P}=\bar{\gamma}_{R D}=\bar{\gamma}_{R P}=Q / \delta_{R}^{2}=$ $Q / \delta_{D}^{2}=\bar{\gamma}$ and ignore higher order terms, we have

$$
P_{\text {out }}^{\infty}\left(\gamma_{0}\right)=\Phi\left(\frac{1}{\bar{\gamma}}\right)^{D}
$$

then the diversity order $D$ and code gain $\Phi$ can be respectively, 


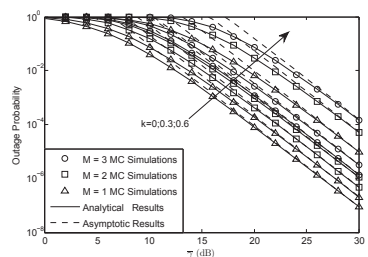

(a) OP versus different $\bar{\gamma}$.

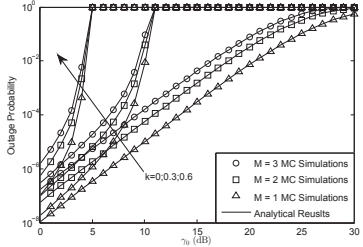

(b) OP versus different $\gamma_{0}$.
Fig. 1. OP of the system

$D=\left\{\begin{array}{l}\text { derived as } \\ D_{1}=N-M+2 \sum_{\xi_{1}=0}^{m_{S P}-1} \cdots \sum_{\xi_{M}=0}^{m_{S P}-1} \Lambda_{S P}, \Phi_{1}>\Phi_{2}, \\ D_{2}=N, \Phi_{1} \leq \Phi_{2},\end{array}\right.$

$\Phi=$

$$
\left\{\begin{array}{l}
\Phi_{1}=\left(\frac{\alpha_{S R}+\alpha_{S R} k_{S P}^{2}}{1-k_{S P}^{2} \gamma_{0}}\right) \sum_{\xi_{1}=0}^{N} \cdots \sum_{\xi_{M}=0}^{m_{S P}-1} \frac{m_{S P}-1}{\left.m_{1}-\delta_{1}\right)\left(\Lambda_{S P}-1+N\right) !} \\
\times \frac{\Xi(M)}{\bar{\gamma}_{S P}^{\left.-2 S_{S P}+\cdots \xi_{M}\right)}} \sum_{\xi_{1}=0}^{m_{S P}-1} \cdots \sum_{\xi_{M}=0}^{m_{S P}-1} \frac{\left(\beta_{1}-\delta_{1}\right)^{M} \Xi(M)\left(\Lambda_{S P}-1+N\right) ! \gamma_{0}^{D}}{\Delta_{S P}^{-\left(\xi_{1}+\cdots \xi_{M}\right)}} \\
\Phi_{2}=\frac{(N+N \times M-1) !}{N !(N \times M-1) !}\left(\frac{1+k_{R P}^{2}}{1-k_{R P}^{2} \gamma_{0}}\right)^{N} \gamma_{0}^{D_{2}} .
\end{array}\right.
$$

\section{NumericAl Results}

In this section, numerical results are provided to validate the performance analysis. The system parameters are given as the GEO, $f=2 \mathrm{GHz}, \varphi_{3 \mathrm{~dB}}=0.8^{\circ}, G_{\max }=48 \mathrm{~dB}$, $G_{r, S J}=4 d B, B=15 \mathrm{MHz}$, and $T=300^{\circ} \mathrm{K}$. Without loss of generality, we set $\delta_{R}^{2}=\delta_{D}^{2}=1$ and in all plots we denote $\bar{\gamma}_{S R}=\bar{\gamma}_{S P}=\bar{\gamma}_{R D}=\bar{\gamma}_{R P}=\bar{\gamma}, k_{1}=k_{2}=k_{S P}=k_{R P}=k$ and $m_{U}=1, b_{U}=0.063, \Omega_{U}=0.0007$. In Fig. 1 (b), we set $\bar{\gamma}=30 \mathrm{~dB}$.

Fig. 1 depicts the OP of the system. It can be derived that the analytical results agree well with the Monte Carlo (MC) results. The asymptotic results are tight across the simulation results at high SNRs, confirming the effectiveness of our performance analysis. In addition, we can observe from Fig. 1(a) that the OP will be degraded with the increase of $M$. This is because a stricter interference constraint is required as $M$ increases. However, the HIs does not affect the diversity order of the CHSTN. Furthermore, we can find that the SNDR would have a ceiling phenomenon in Fig. 1(b), namely, when $\gamma_{0}$ is larger than a specific value, the OP would be always 1 as $\gamma_{0}$ grows to infinity. In this case, the existence of HIs determines an extreme values of $\gamma_{0}$ where an outage is inevitable. Besides, the ceiling effect is just the function of the aggregate HIs level, and the system performance will be worse when the aggregate level of HIs is larger.

\section{CONCLUSIONS}

In this paper, we have investigated the OP of a cognitive hybrid satellite-terrestrial networks with HIs and multi-primary users. Specifically, the closed-form expressions of the OP for the system have been derived. To get further insights, we have derived the asymptotic expressions of OP at high SNRs. Our works provides an efficient way to evaluate the impact of nonideal hardware on the performance of cognitive hybrid satellite networks with multi-primary users.

\section{REFERENCES}

[1] M. A. Vazquez, A. Perez-Neira, et al., "Precoding in multibeam satellite communications: present and future challenges," IEEE Wireless Commun., vol. 88, no. 95, pp. 4390-4404, Dec. 2016.

[2] M. K. Arti, and S. K. Jindal, "OSTBC transmission in shadowed-Rician land mobile satellite links," IEEE Trans. Vehi. Tech., vol. 65, no. 7, pp. 5771-5777, Jul. 2016.

[3] R. B. Manav and M. K. Arti, "On the closed-form performance analysis of maximal ratio combining in shadowed-Rician fading LMS channels," IEEE Commun. Lett., vol. 18, no. 1, pp. 54-57, Jan. 2014.

[4] S. K. Sharma et al., "Cognitive radio techniques for satellite communication systems," in Proc. IEEE VTC-Fall, 2013, pp. 1-5.

[5] S. Vassaki, M. I. Poulakis, A. D. Panagopoulos, et. al., "Power allocation in cognitive satellite terrestrial networks with QoS constraints," IEEE Commun. Lett., vol. 17, no. 7, pp. 1344-1347, Jul. 2013.

[6] K. An, M. Lin, W. Zhu, Y. Huang, and G. Zheng, "Outage performance of cognitive satellite terrestrial networks with interference constraint," IEEE Trans. Vehi. Tech., vol. 65, no. 11, pp. 9397-9404, Jan. 2016.

[7] K. An, et al., "Secure transmission in cognitive satellite terrstrial networks," IEEE J. Sel. Area, vol. 34, no. 11, pp. 3025-3037, Nov. 2016.

[8] P. K. Sharma, P. K. Upadhyay, D. B. costa, et. al., "Performance analysis of overlay spectrum sharing in hybrid satellite-terrestrial systems with secondary network selection," IEEE Trans. Wire. Commun., vol. 16, no. 10, pp. 6586-6601, Oct. 2017.

[9] K. An, J. Ouyang, M. Lin, and T. Liang, "Outage analysis of multiantenna cognitive hybrid satellite-terrestrial relay networks with beamforming," IEEE Commun. Lett., vol. 19, no. 7, pp. 1157-1160, Jul. 2015.

[10] A. Guidotti, et.al., "Satellite-enabled LTE systems in LEO constellations," IEEE 2017. ICC., May. 2017. Pairs, France, pp. 876-881.

[11] O. Kodheli, A. Guidotti, A. Vanelli-Coralli, "Integration of Satellites in 5G through LEO Constellations," IEEE Globecom 2017., Dec. 2017, Singapore, Singapore, pp. 1-6.

[12] S. Chatzinotas, et.al., "Cognitive Approaches to Enhance Spectrum Availability for Satellite Systems," International Journal of Satellite Communications and Networking, vol. 35, no. 5, pp. 407,442, Nov. 2016

[13] E. Costa, and S. Pupolin, "M-QAM-OFDM system performance in the presence of a nonlinear amplifier and phase noise," IEEE Trans. Commun., vol. 50, no. 3, pp. 462-472, Mar. 2002.

[14] T. Schenk, "RF imperfections in high-rate wireless systems: impact and digital compensation", Springer, Netherlands, 2008, pp. 2033-2043.

[15] E. Bjornson, M. Matthaiou, and M. Debbah, "A new look at dual-hop relaying: Performance limits with hardware impairments," IEEE Trans. Commun., vol. 61, no. 11, pp. 4512-4525, Nov. 2013.

[16] N. I. Miridakis, D. D. Vergados, and A. Michalas, "Cooperative Relaying in Underlay Cognitive Systems with TAS/MRC, Spatial Correlation and Hardware Impairments," IEEE Vehicular Technology Conference (VTC), Boston, USA, Sep. 2015.

[17] N. I. Miridakis, D. D. Vergados, and A. Michalas, "Cooperative relaying in underlay cognitive systems with hardware impairments," $A E U$ International Journal of Electronics and Communications, vol. 69, no. 12, pp. 1885-1889, Dec. 2015.

[18] "8 hints for making and interpreting EVM measurements," Tech. Rep., Agilent Technologies, Santa Clara, CA, USA,5989-3144EN, 2005.

[19] P. K. Sharma and P. K. Upadhyay, "Cognitive relaying with transceiver hardware impairments under interference constraints," IEEE Commun. Lett., vol. 20, no. 4, pp. 820-823, Apr. 2016.

[20] G. Zheng, P D. Arapoglou and B. Ottersten, "Physical layer security in multibeam satellite systems," IEEE Trans Wire. Commun., vol. 11, no. 2, pp. 852-863, Feb. 2012.

[21] S. K. Sharma, S. Chatzinotas, and B. Ottersten, "Transmit beamforming for spectral coexistence of satellite and terrestrial networks," in Proc ICCROWN, Jul. 2013, Washington, DC, USA, pp. 275-281.

[22] N. I. Miridakis, D. D. Vergados, and A. Michalas, "Dual-hop Communication over a Satellite Relay and Shadowed Rician Channels," IEEE Trans. Vehi. Tech., vol. 64, no. 9, pp. 4031-4040, Sep. 2015.

[23] I. S. Gradshteyn, I. M. Ryzhik, A. Jeffrey, et. al., "Table of integrals, series and products," 7thed.Amsterdam, Boston: Elsevier, 2007.

[24] A. Bletsas, H. Shin, and M. Z. Win, "Cooperative communication with outage-optimal opportunistic relaying," IEEE Trans. Wire. Commun., vol. 6, no. 9, pp. 3450-3460, Sep. 2007. 